\title{
Be Envelope Properties Determined from Fe II Emission Lines $^{1}$
}

\author{
J. Chauville, D. Ballereau
}

Observatoire de Paris-Meudon, DASGAL/UMR8633 du CNRS, F-92195 Meudon Principal Cedex, France

L. Cidale, N. Morrell

Observatorio Astronómico de La Plata, Paseo del bosque S/N, 1900 La Plata, Argentina

\section{Friedjung, J. Zorec}

Institut d'Astrophysique de Paris, CNRS, $98^{\text {bis }}$ Bd. Arago, F-75014 Paris, France

\begin{abstract}
Applying the SAC (self absorption curve) method to $\chi$ Oph (HD 148184) we conclude that FeII emission lines are optically thick. We estimate the excitation temperature of the permitted Fe II line formation region and the dimensions of this region. We conclude that this region may possibly have a non-uniform density structure.
\end{abstract}

\section{Observations}

The spectrum of $\chi$ Oph studied in this paper was obtained on 5 March 1990 with the $2.15 \mathrm{~m}$ telescope at the CASLEO Observatory in Argentina, using a Cassegrain REOSC type SE spectrograph and a TEK 1024x1024 pix CCD detector. The observed spectral range is $\lambda \lambda 4900-9000 \AA$ and the spectrum has a resolution $\mathrm{R}=11500$ and $\mathrm{S} / \mathrm{N}=450$.

\section{Study of Fe II lines}

In view of the abundance of $\mathrm{Fe}$ II emission lines in the spectrum, it is possible to study the properties of their region of formation, using the "self absorption curve" (SAC) method (Friedjung \& Muratorio 1987, Muratorio \& Friedjung 1988, Muratorio et al. 1992). This method independently gives information about the populations of the upper and lower terms of the spectral lines, as long as deviations from LTE are almost constant for the levels inside the same term. This is not possible with classic emission line curve of growth methods. In fact,

\footnotetext{
${ }^{1}$ Data obtained in CASLEO operated under agreement between the CONICET and the Universities of La Plata, Córdoba and San Juan - Argentina
} 


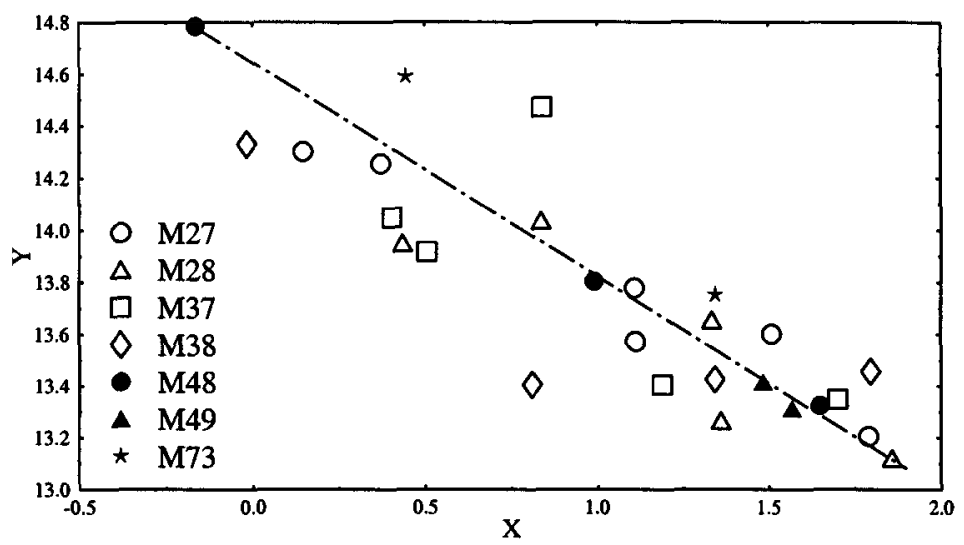

Figure 1. The SAC determined by several multiplets of FeIl emission lines produced in the circumstellar envelope of $\chi$ Oph. The slope of the SAC indicates that the FeII emission lines are optically thick.

the flux integrated over a spectral line is given by:

$$
Y=\log \left[F_{\mathrm{ul}} \lambda^{3} / g_{l} f_{\mathrm{lu}}\right]=Q(\tau)+\log \left[N_{\mathrm{u}} / g_{\mathrm{u}}\right]+H
$$

Here $\log F_{\mathrm{ul}}$ is the line flux, $g_{\mathrm{l}}$ is the lower level statistical weight, $f_{\mathrm{lu}}$ is the oscillator strength, $N_{\mathbf{u}}$ is a characteristic upper level column density, $g_{\mathbf{u}}$ the upper level statistical weight and $H$ is a geometrical factor. $Q(\tau) \simeq \log [(1-$ $\left.\left.e^{-\tau}\right) / \tau\right]$ is the SAC function, which depends on the optical depth $\tau$ of the line in the formation region. The opacity $\tau$ depends on deviations from homogeneity in the line formation region. $H$ depends on the distance, the radius of the line emitting region and a characteristic line width.

The line opacity $\tau$ is related to $X=\log g_{1} f \lambda$, so that:

$$
X=\log \tau-\log \left[N_{1} / g_{1}\right]+\text { const., }
$$

with $N_{\mathrm{l}}$ the characteristic lower level column density. Graphs of $X$ against $Y$ can be drawn for different multiplets with different upper and lower terms, so relative shifts give relative upper and lower term populations and therefore excitation temperatures when populations are not too far from a Boltzmann distribution. The upper metastable levels of the usually optically thin forbidden Fe II lines $[Q(\tau)=0$ in eq. (1)] are lower terms of optical permitted lines. Hence, the relative populations of the same terms can be found assuming line formation in the same region.

In Fig. $1 \mathrm{Y}$ is shown as a function of $\mathrm{X}$ for Fe II line multiplets of our object having lower terms with almost the same excitation potential. The slope $\partial Y / \partial X<0$ indicates that the plotted transitions are optically thick in all regions of line formation. For a very large optical thickness in all such regions of line formation, the slope tends to -1 . 


\section{Results obtained}

One of the most interesting advantages of the SAC method is that it allows us to determine the excitation temperature of upper and lower levels of the Fe II emission lines studied. These temperatures then lead to an estimate of the extent of the envelope region producing the emission lines. Data gathered in this poster have not yet been examined in enough detail to proceed to make vertical and horizontal displacements of individual SACs of multiplets needed for temperature determinations. We shall however give a rough estimate of the mean excitation temperature of the Fe II lines and of the radius of the region in the circumstellar envelope (CE) which produces them. In order to do so, we need to discuss the visible flux excess produced by the CE.

(a) Extent and mean temperature of the $C E$ region producing the visible continuum excess

From the BCD (Paris spectrophotometric spectral classification system) data we deduce the following fundamental parameters for $\chi$ Oph: $T_{\text {eff }}=23000$ $\mathrm{K}, \log g=4.0 ; R / R_{\odot}=5.2$.

Using the techniques developed in Moujtahid et al. (1999), we find from spectrophotometric observations made simultaneously to the spectroscopic ones that the region in the CE producing the visible continuum flux excess has: (1) a total optical depth at $\lambda 0.56 \mu \mathrm{m} \tau_{\mathrm{V}}=\tau_{(b f+f f)}+\tau_{\mathrm{e}} \simeq 1.1$; (2) a mean electron temperature $\overline{T_{\mathrm{e}}} \simeq 10200 \mathrm{~K}$, derived assuming that the medium is in LTE; (3) an extent of $R / R_{*} \simeq 2.3$.

(b) Mean excitation temperature of Fe II lines and extent of the region producing them

Relation (1) between the SAC curve $\mathrm{Q}(\tau)$ and the observed parameters of Fe II lines which allows us to calculate the extent $R_{\mathrm{c}}$ of the emitting region can be rewritten as:

$$
\left(\frac{R_{\mathrm{c}}}{d}\right)^{2}=\frac{1}{2 \pi h c}\left[\frac{10^{-Q(\tau)}}{\tau}\right] \times \frac{F_{\mathrm{c}} W_{\lambda} \lambda^{4}}{V_{\mathrm{c}}} \times \mathrm{e}^{1.44 / \lambda T_{\mathrm{r}}}
$$

where $\tau$ is the optical depth of the lower level for a given $\lambda$ line whose equivalent width is $W_{\lambda} ; F_{\mathrm{c}}$ is the local continuum flux; $v_{\mathrm{c}}$ is a typical velocity parameter (we used the half-width at half emission intensity of lines); $d$ is the stellar distance ( $d$ $=150 \mathrm{pc}$ HIPPARCOS); $T_{\mathrm{r}}$ is the excitation temperature. Equation (3) assumes a $\mathrm{Q}(\tau)$ characteristic of a uniform slab with a constant source function.

Fig. 1 shows that the CE of $\chi$ Oph is optically thick for permitted Fe II lines. To estimate the order of magnitude of some CE parameters, we can then assume that $\tau \simeq 1$ because the slope of the SAC is between 0 and -1 . Hence, (3) gives a relation between $R_{\mathrm{c}}$ and $T_{\mathrm{r}}$ for each multiplet. As these relations are not too different for the multiplets plotted in Fig. 1, we adopted a mean relation which can be recast into the following expression:

$$
\frac{T_{\mathrm{r}}}{\overline{\bar{T}_{\text {eff }}}}=0.15+0.51 \times\left(\frac{R_{*}}{R_{\mathrm{c}}}\right)
$$


which can be compared with a similar one obtained by Moujtahid et al. (1999) between $\overline{T_{\mathrm{e}}}$ and the extent of the region producing the continuum flux excess. Both relations then lead to:

$$
T_{\mathrm{r}} \simeq 1.1 \times \overline{T_{\mathrm{e}}}-3700
$$

which is independent of $R$ as long as the line and continuum forming regions are common. At $R>2.3 R_{*}$ the visible continuum is optically thin, while the Lyman continuum is still in detailed balance for the next few radii and the CE temperature can be assumed not to change significantly at $R>2.3 R_{*}$. So, using (4), (5) and the parameters deduced in (a) we obtain for the Fe II emission line forming region:

$$
\begin{gathered}
T_{\mathrm{r}} \sim 7500 \pm 600 \quad \mathrm{~K} \\
R_{\mathrm{c}} \sim 3.0 \pm 1.0 R_{*}
\end{gathered}
$$

\section{Conclusions}

Our results are somewhat preliminary and need to be confirmed by more detailed analysis, which we shall be undertaking. This kind of study will be carried out for about 100 classical Be stars we have already observed. One point which should also be noted is that the slope of the SAC is of the order of -0.7 over a large range of $\log g f \lambda$. This can be understood if column densities are not the same over lines of sight to different regions of line formation, that is if the envelope has a non-uniform density structure.

\section{References}

Friedjung, M., \& Muratorio, G. 1987, A\&A 188, 100

Moujtahid A., Zorec J., Hubert A.M. 2000, this volume: "On the characteristics of the CE near the central object"

Muratorio, G., \& Friedjung, M. 1988, A\&A 190, 103

Muratorio, G., Viotti, R., Friedjung, M., Baratta, G.B., Rossi C. 1992, A\&A 258,423 